\title{
A Newly Improved Canny Algorithm of Image Edge Detection
}

\author{
Xiang Wu ${ }^{1, a}$, Weibo $\mathrm{Yu}^{{ }^{*} 1, \mathrm{~b}}$ Xiaotong Liu $^{1}$ Keping Liu $^{1}$ \\ ${ }^{1}$ School of Electrical \& Electronic Engineering, Changchun University of Technology, Changchun, \\ 130012, China \\ aemail:wu_xiang2015@126.com, bemail: yu_weibo@126.com
}

Keywords: Edge detection; Median filtering; Gradient amplitude; Canny Detector

\begin{abstract}
A new adaptive Canny operator is proposed which aims at the effect of image edge areas when traditional Canny operator under Gaussian smooth and the shortcomings of threshold level need to be pre-set. Firstly, the adaptive median filtering is adopted to process image; Secondly, this paper adds the gradient template in the direction of $45^{\circ}$ and $135^{\circ}$ to calculate gradient amplitude; Finally, get the high and low threshold value of images by calculating gradient amplitude operation with the Otsu algorithm. In this paper, a series of experiments are designed and it verities the validity of the improved algorithm. The experiment results show that the improved Canny algorithm has a good anti-noise performance, and it can accurately detect the edge information. It reduces the existence of false edge and enhances the practicality of the algorithm.
\end{abstract}

\section{Introduction}

Image edge information is one of the most important information in an image, which can be described the target outline, the relative position within the target area, and other important information. Along with the development of computer vision and digital image processing technology, edge detection is widely used in all aspects of life, especially in the detection of the size of work piece, edge detection is an essential step. Most traditional edge detection methods take operation on the neighbor region pixels, and obtain the gradient with templates approximation, such as Prewitt, Robert, and Sobel, which are relatively simple and easy to implement, and it has a good real-time performance, but these operators are sensitive to noise, poor anti-interference performance, and their detection effect are not perfect in engineering application [1]. Compared with these edge detection algorithms, Canny operator is more accurate, and it has become the standard of evaluation of other edge detection method [2][3]. However, the traditional Canny algorithm adopts fixed spatial scale parameters for image smoothing. The high and low threshold of edge detection is lack of adaptability for different images. What' $\mathrm{s}$ more, the traditional Canny uses $2 \times 2$ template to calculate the gradient, it is more sensitive to noise. Using iterative method to calculate the best threshold as the high-low threshold in literature [4], the improved algorithm has no obvious advantages to the noisy images and large scale of calculation, so it needs a long time and the results are poor. Literature[5] directly uses Canny Otsu algorithm of edge detection, which is easy detect of the false edges, not conducive to practical applications. Therefore, the paper proposes the improved Canny operator edge detection, the experiment results show that this given method has satisfactory results compared with the traditional Canny operator and the method in reference [5].

\section{The Basic Principle and Analysis of the Traditional Canny Algorithm}

In 1986, John F. Canny proposed three criteria to judge image edge detection operator' performance: SNR criterion, localization precision criterion and single edge response criterion [6].

\section{The Traditional Canny Algorithm.}

The traditional canny image edge detection is divided into the following steps: the first step of traditional Canny algorithm is to smooth the original image by using a Gaussian filter, calculate the gradient magnitude and gradient direction; the second step is to adopt non-maximal inhibition after

* Corresponding author, E-mail: yu_weibo@126.com 
pretreatment of gradient image histogram, to refine those edges, and finally use fixed threshold to reduce the false edge points and to connect edges [7].

1) image filtering

Choose appropriate 1-d Gaussian function to smooth the image, according to the row and column respectively [8], the Gaussian filter function (as shown in (1)) to smooth image and remove noise.

$$
G(x)=\frac{1}{\sqrt{2 \pi \sigma}} \exp \left(-\frac{x^{2}}{2 \sigma^{2}}\right)
$$

Where $\sigma$ is constant of space, control the degree of image smoothing.

2) calculate gradient amplitude and direction of image

Next step is to calculate the magnitude and the direction of image gradient. The traditional Canny algorithm adopts limited difference of $2 \times 2$ neighboring area to calculate the value and direction of image gradient. The first order partial derivative of $\mathrm{X}$ direction and $\mathrm{Y}$ direction can be got from these following formulas:

$$
\left\{\begin{array}{l}
P_{x}(x, y)=[f(x, y+1)-f(x, y)] / 2+[f(x+1, y+1)-f(x+1, y)] / 2 \\
P_{y}(x, y)=[f(x, y)-f(x+1, y)] / 2+[f(x, y+1)-f(x+1, y+1)] / 2
\end{array}\right.
$$

Among them, position relationship between pixels as shown in table 1.

Table 1 pixel location relational tables

\begin{tabular}{cc}
\hline$f(x, y)$ & $f(x, y+1)$ \\
\hline$f(x+1, y)$ & $f(x+1, y+1)$ \\
\hline
\end{tabular}

Note: the grey value represants point in the table, such as point $(x, y)$ grey value is $f(x, y)$

The gradient amplitude can be determined as:

$$
P(x, y)=\sqrt{P_{x}(x, y)^{2}+P_{y}(x, y)^{2}}
$$

The azimuth of the image gradient is:

$$
\theta(x, y)=\arctan \left[\frac{P_{y}(x, y)}{P_{x}(x, y)}\right]
$$

3) non-maxima suppression (NMS)

Apply the NMS on the image to accurately position edges. The process of the NMS can help guarantee that the single-pixel position and retain the maximum amplitude of local variation of the point, which is non-maxima suppression treatment [9]. In the $3 \times 3$ neighboring area, the center pixel and its adjacent two pixels are compared, if greater than, the point is an edge point; if less than, the point is non-edge point.

4) thresholding and edge connection

Canny algorithm uses dual-threshold $T_{h}$ and $T_{l}$ method to select edge points after carrying on non-maximum suppression over the image segmentation. If $(x, y)$ less then $T_{l}$, then this point is not the edge point; If the $(x, y)$ gradient magnitude greater than $T_{h}$, the point must be the edge points; if $P(x, y)$ in between $T_{l}$ and $T_{h}$, then On the search $(x, y)$ in the neighboring area of the point is greater than $T_{h}$,if there is, this is a point, otherwise, it is not the edge.

\section{The Defect of the Traditional Canny Operator.}

Compared with other traditional edge detection algorithms, the traditional Canny algorithm has better detection effect, but also it has many shortcomings, such as the traditional Canny algorithm adopts first order limited different $2 \times 2$ neighboring area to calculate image's gradient. It's is easy to calculate, but it's more sensitive to noise, which is easy lose the image edge. The Gauss variance and dual threshold are manually set, for the rich image edge information, the adaptive ability is poor, and it's very easy lose detail information. To solve these problems, the paper proposes the improved the Canny operator image the edge detection, the method could effective to move the noise and get a better image processing results. 


\section{Improved Canny Edge Detection Algorithm}

Based on the traditional Canny algorithm, this paper adopts the adaptive median filter and change the method of calculating the gradient amplitude, the threshold calculating algorithm has been improved.

\section{Adaptive Median Filter.}

The first step is to smooth image, the traditional Canny edge detector is Gaussian smoothing function, the variance of the Gauss function needs to be set artificially, and the Gauss filter will blur the image, which may lead to image filtered not meet the requirements. In this paper, the adaptive median filter is used to improve the first step of the Canny algorithm and it can effectively protect the image edges.

The adaptive median filter by changing the size of the template window, by different processing methods deal with the noise points and the signal points, so as to achieve the good effect of removing the noise [10]. when carrying out de-noising, the adaptive median filter algorithm is used to change the size of the template window, so that the filter output a single value, the value is used to replace the template window center point $(x, y)$ pixel value.

For a size of image $M \times N$, setting $S_{x y}$ as the working window of the current pixel $(x, y)$ de-noising processing, $S_{\max }$ is the maximum filtering window; $f(x, y)$ is the gray value of the pixel point $(x, y) ; f_{\min }$ is the minimum gray value of pixels; $f_{\max }$ is the maximum gray value; $f_{\operatorname{med}}$ is the median.

The specific implementation steps of the adaptive median filter are as follows:

Step 1: Initialize the size of filter window $w=3$;

Step 2: Calculate the minimum $f_{\min }$, maximum $f_{\max }$, median $f_{\text {med }}$ value of the gray in the current template window.

Step 3: If $f_{\min }<f_{\operatorname{med}}<f_{\max }$, then turn to Step 5; Otherwise increase the size of window, $w=w+2$;

Step 4: If $w \leq S_{\max }$, then turn to Step 2; Otherwise use templates window median $f_{\text {med }}$ instead of the current pixel, namely $f(x, y)=f_{\text {med }}$;

Step 5: If $f_{\min }<f(x, y)<f_{\max }$, it indicates that the point is not a noise point, the filter output is still $f(x, y)$; Otherwise, $f(x, y)$ is replaced by $f_{\text {med }}$, namely $f(x, y)=f_{\text {med }}$.

\section{Improved Gradient Magnitude Calculation.}

The traditional Canny algorithm uses the limited different of $2 \times 2$ neighboring area to calculate the image's gradient, this method is more sensitive to noises. In $3 \times 3$ window add $45^{\circ}$ and $135^{\circ}$ direction gradient amplitude calculation, this method can reduce the interference of noise, but the computational complexity and low efficiency in the literature [11]. On the basis of Sobel operator, this paper adds the gradient template of $45^{\circ}$ and $135^{\circ}$ to calculate gradient amplitude. Four templates direction as showed in Fig.1.

\begin{tabular}{|c|c|c|}
\hline-1 & -2 & -1 \\
\hline 0 & 0 & 0 \\
\hline 1 & 2 & 1 \\
\hline
\end{tabular}

(a) $\mathrm{x}$ axis direction

\begin{tabular}{|c|c|c|}
\hline-2 & -1 & 0 \\
\hline-1 & 0 & 1 \\
\hline 0 & 1 & 2 \\
\hline
\end{tabular}

(c) $45^{\circ}$ direction

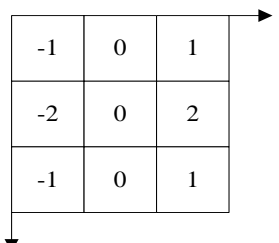

(b) y axis direction

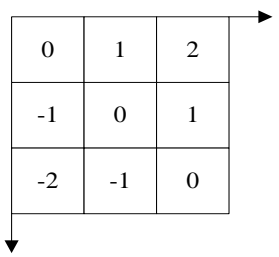

(d) $135^{\circ}$ direction

Fig.1 Gradient template 
Gradient component of the four directions: $P_{x}(x, y), P_{y}(x, y), P_{45^{\circ}}(x, y), P_{135^{5}}(x, y)$ can be determined by convolution with of figure 2 templates respectively. Gradient magnitude can be obtained from the above four components. the image gradient magnitude is:

$$
\begin{aligned}
& P(x, y)=\sqrt{c(x, y)} \\
& \text { among them: } C(x, y)=P_{x}(x, y)^{2}+P_{y}(x, y)^{2}+P_{45^{\circ}}(x, y)^{2}+P_{135^{\circ}}(x, y)^{2}
\end{aligned}
$$

\section{The Improvement of Threshold Value Algorithm.}

Since the parameters of the traditional Canny algorithm are fixed, which leads to the algorithm low efficiency and cannot adapt to different situations. Adapting Otsu algorithm to calculate the gradient image threshold $T_{h}$, at the same time set $T_{l}=T_{h} / 2$. Improved algorithm enhances the Canny algorithm of adaptive ability.

Otsu [12] method was proposed by Otsu in 1980. In this method, the gray histogram of the image is used to measure the target and the background of the inter class variance. The optimal threshold value is obtained when the value of the measure criterion function is taken to obtain the maximum value [13]. Otsu method is widely used because it is simple and does not require a prior knowledge.

If an image has $n$ pixel points, the number of gray value for $i$ is $n_{i}$, then the probability of $i$ is $P_{i}=n_{i} / n$. the image is divided into two classes $C_{1}=[1,2, \cdots, k]$ and $C_{2}=[k+1, k+2, \cdots, n]$ by threshold $k$, the probability of $C_{1}$ and $C_{2}$ are $\omega_{1}=\sum_{i=1}^{k} P_{i}$ and $\omega_{2}=\sum_{i=k+1}^{n} P_{i}=1-\omega_{1}$, respectively corresponding to the average are $\mu_{1}=\sum_{i=1}^{k} i P_{i} / \omega_{1}$ and $\mu_{2}=\sum_{i=k+1}^{n} i P_{i} / \omega_{2}$. Suppose $\mu_{T}=\sum_{i=1}^{n} i P_{i}$ is the whole average gray level, then the formula of variance between $C_{1}$ and $C_{2}$ as shown in equation (5).

$$
\sigma^{2}=\omega_{1}\left(\mu_{1}-\mu_{T}\right)^{2}+\omega_{2}\left(\mu_{2}-\mu_{T}\right)^{2}=\omega_{1} \omega_{2}\left(\mu_{2}-\mu_{1}\right)^{2}
$$

As known from the analysis of the above, the best threshold is obtained when $\sigma^{2}$ is maximum, and the image segmentation effect is the best.

The specific procedures of the improved Canny edge detection algorithm are as follows:

Step 1: Acquire image (Lena and gear).

Step 2: Smooth filtering (adaptive median filter).

Step 3: Calculate gradient amplitude and direction of image

Step 4: Non-maxima suppression (NMS).

Step 5: Determine the high and low threshold, detect and connect edges.

Step 6: Get the edge image.

\section{The Results of the Experiments and Analysis}

To verify the effectiveness of the improved algorithm, we designed two groups of experiments. The program language we used to run the image process program is MATLAB2010a.

The first group of experiments selects the standard Lena gray as the test image, which is a size of $256 \times 256$. The results of the image edge detection are shown in Fig.2.

From Fig.2(b) and Fig.2(c), we can see that there is a clear fracture in the face and cap. A fracture to the left of the pole, the details of the hair is not very good. From Fig.2 (d) we can get the image edge is clearer and more precise. Obviously, the effect of improved the Canny algorithm is better in the integrity of the edges.

The second group of selects the $598 \times 848$ no standard gear gray image. The results of gear edge detect are showed in Fig.3. It has a lot of fake edges (due to the setting of threshold too higher) and is not clear in Fig.3 (b). Compared Fig3.(c) with Fig.3(d), the mentioned in the literature five and improved Canny algorithm in this paper can well detect the gear edge and keep the edge information of the image, but the improved algorithm detect the edge more effectively and the detection results are satisfied. 


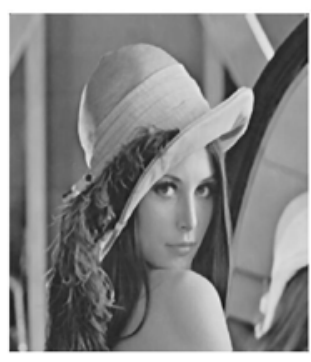

(a)

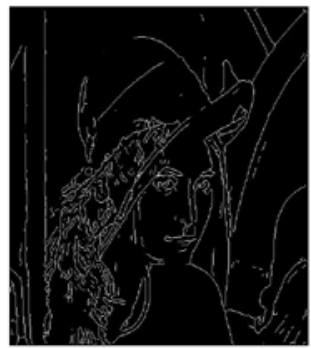

(c)

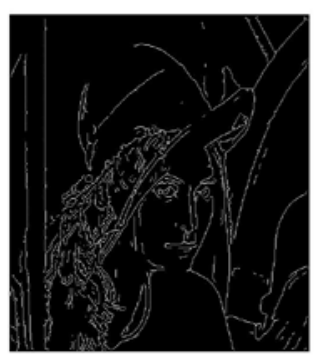

(b)

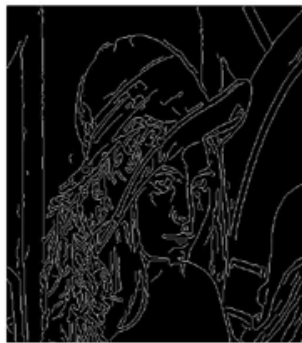

(d)

Fig.2 Comparison of edge detection results for Lena image (a) noise image. (b) traditional Canny algorithm. (c) mentioned in the literature five. (d) improved Canny algorithm in this paper.

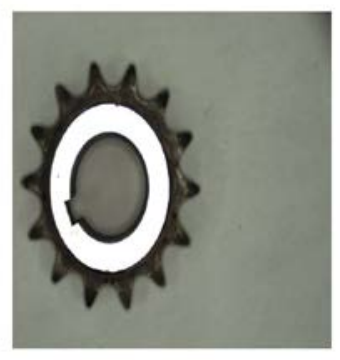

(a)

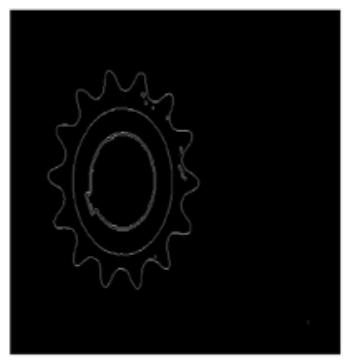

(c)

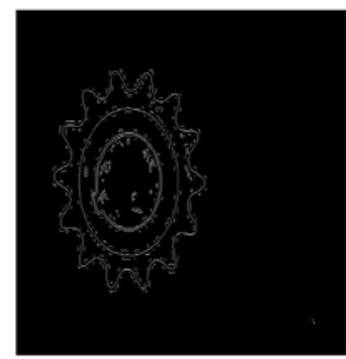

(b)

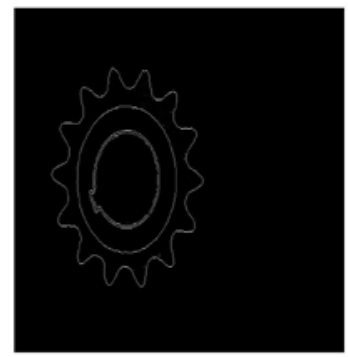

(d)

Fig.3 Comparison of edge detection results for industrial part image (a) gear image. (b) traditional Canny algorithm. (c) mentioned in the literature five. (d) improved Canny algorithm in this paper.

\section{Conclusions}

The traditional Canny algorithm has difficulty in processing images which contains the noise, and it needs to manually set threshold that the adaptive ability is poor in the variance of the Gaussian filtering. For this problem, a new Canny algorithm is presented in this paper, in which uses the adaptive median filter treat the noise image, and the gradient magnitude is calculated by adding gradient template the directions of $45^{\circ}$ and $135^{\circ}$. Finally, adopt the method of the Ostu to calculate the image's threshold $T_{h}$, as the same the time suppose $T_{l}=T_{h} / 2$. Experimental results show that the improved algorithm not only keeps the advantages of the traditional Canny algorithm, but also it enhances the ability of noise suppression and ensures the accuracy and precision of edge 
detection. the improved algorithm can obtain the threshold automatically, which has higher practical engineering application.

\section{Acknowledgement}

In this paper, the research was sponsored by the Jilin Provincial Science and Technology Development Project (Project NO. 20140204018GX) and Jilin Provincial Youth Leading Talent and Team Project of Science and Technology Innovation (Project NO. 20150519009JH).

\section{References}

[1] Zhang Fan, Peng Zhong-wei and so on. Improved Canny edge detection method based on self-adaptive threshold. Journal of Computer Applications, 2012, 32(8): 2296-2298.

[2] J. Canny. A computational approach to edge detection. IEEE Transactions on Pattern Analysis and Machine Intelligence, 1986, 8(6): 679-698.

[3] K A. Panetta, E J. Wharton, S S. Agaian. Logarithmic edge detection with applications. Journal of Computers, 2008, 3(9): 3346-3351.

[4] Wang Xiao-jun, Liu Xu-min and so on. Image Edge Detection Algorithm Based on Improved Canny Operator .Computer Engineering, 2012, 38(14): 196-198+202.

[5] F Mei, G X. Yue , Q C. Yu. The study on An Application of Otsu method in canny operator//ISIP’09, Huangshan, China, August21-23, 2009: 109-112.

[6] Zhang Zhen and so on. Improved Image Edge Extraction Algorithm Based on Canny Operator. Journal of Jilin University (Science Edition), 2007, 45(2): 224-248.

[7] Xu Hong-ke and so on. An Improved Algorithm for Edge Detection Based on Canny. Infrared Technology, 2014, 36(3): 210-214.

[8]Lulu Tang , Qican. Zhang and so on. An Improved Algorithm for Canny Edge Detection with Adaptive Threshold. Opto-Electronic Engineering, 2011, 38(5):127-132.

[9] Hengyang Su , Xianzhen Yuan. An Improved Canny Edge Detection Algorithm. Computer Simulation, 2010, 27(10):242-245.

[10] Wang Xiao-kai, Li Feng. Improved adaptive median filtering. Computer Engineering and Applications, 2010, 46(3): 175-176.

[11] Junshan Li and so on. A Novel Arithmetic of Image Edge Detection of Canny Operator. Acta Photonica sinica, 2011, S1:50-54.

[12] Otsu. N. A threshold selection method from gray-level histograms . IEEE Transactions on Systems. Man and Cybernetics, SMC29,1979, 9(1):62-66.

[13] Yang Hao, Pei Lei, Changshun Li. Fast algorithm of subpixel edge detection based on Zernike moments. Application Research of Computers, 2011,28(11):4380-4382+4385. 